\title{
Avaliação de fatores de risco psicossociais: estudo com docentes do ensino superior
}

\author{
Psychosocial risks evaluation factors: Study with higher education teachers
}

Artigo Original | Original Article

Sara Lopes Borges, PhD (1a), Cristina Santos, PhD (2b), António Saraiva, PhD (2b), Margarida Tenente Pocinho, PhD (2c)

(1) Instituto Superior Miguel Torga, Coimbra, Portugal

(2) Escola Superior de Tecnologias da Saúde de Coimbra - Instituto Politécnico de Coimbra, Portugal

(a) Revisão da literatura, Interpretação e Discussão dos resultados, Redação do manuscrito.

(b) Conceção e planeamento do estudo, Recolha de dados e Análise Estatística.

(c) Revisão do Manuscrito e Análise Estatística.

Autor para correspondência | Corresponding author: Sara Lopes Borges; saralopesborges@ismt.pt

Palavras-Chave

Docentes de ensino superior

Fatores de risco psicossocial

\section{RESUMO}

Objetivos: Os fatores psicossociais, enquanto características relacionadas com condições e organização do trabalho, interferem na saúde dos trabalhadores, e os riscos psicossociais emergem da interação entre colaboradores e condições de vida e de trabalho. O objetivo do estudo foi avaliar os fatores de riscos psicossociais em docentes do ensino superior de modo a perceber o exercício da docência enquanto profissão de risco em termos de esgotamento físico e mental, dada a sua contínua exposição a situações risco psicossocial.

Método: O estudo contou com a administração de dois instrumentos, um de caracterização da amostra e o outro para avaliar fatores de risco psicossociais - a versão portuguesa do Copenhagen Psychosocial Questionnaire — constituído por 76 itens (escala tipo Likert de 5 pontos), distribuídos por cinco dimensões, que medem indicadores de exposição a riscos psicossociais e os seus efeitos.

Resultados: No estudo participaram 59 docentes, a maioria homens (50,8\%), com idade entre os 41 e os 50 anos (45,8\%), mestres (59\%), professores adjuntos (47,5\%), com vínculo laboral estável (68\%) e a lecionar entre 11 e 17 horas semanais $(64,4 \%)$. A análise das várias subescalas revelou risco psicossocial, mostrando que os docentes se encontravam em situação de vulnerabilidade. Existiram diferenças significativas entre os riscos vivenciados no ensino superior público e os experimentados no ensino superior privado. O sexo, idade, formação académica e categoria profissional influenciaram o tipo de risco psicossocial.

Conclusões: Confirma-se a importância da avaliação dos fatores de risco psicossociais no exercício da profissão docente no ensino superior. Reconhece-se como necessária a avaliação e gestão dos riscos psicossociais de forma a promover condições de trabalho saudáveis, garantir respeito e tratamento justo, bem como incentivar a promoção da conciliação da vida profissional e familiar, de modo a minimizar riscos psicossociais e situações de vulnerabilidade em docentes do ensino superior.

\section{ABSTRACT}

Objectives: The psychosocial factors as characteristics related to the conditions and organization of work, interfere with the health of employees, and the psychosocial risk situations emerge from the interactions between the employees and their life and work conditions.

The objective of the study was to evaluate the psychosocial risk in higher education teachers in order to perceive the teaching profession as a risk profession in terms of physical and mental exhaustion, given their continued exposure to psychosocial risk situations.

Method: The study consisted of the administration of two instruments, one for the characterization of the sample and the other for assessing psychosocial risk factors - the Copenhagen Psychosocial Questionnaire - consisting of 76 items (5point Likert scale), distributed in five dimensions, which measure indicators of exposure to psychosocial risks and their effects.

Results: The study included 59 teachers, mostly men (50.8\%), aged between 41 and 50 years (45.8\%), with a master's degree $(59 \%)$, assistant professors (47.5\%); with a stable employment relationship (68\%), and teaching between 11 and 17 hours a week (64.4\%). The analysis of the various subscales revealed a psychosocial risk, showing that teachers are in a situation of vulnerability. There were significant differences between the risks experienced in public higher education and those experienced in private higher education. Sex, age, academic background, and professional category influenced the type of psychosocial risk.

Conclusions: The study confirms the importance of the evaluation of psychosocial risk factors in the exercise of the teaching profession in higher education. It is recognized that it is necessary to assess and manage psychosocial risks in order to promote healthy working conditions, ensure respect and fair treatment, and encourage the promotion of work / family life balance, in order to minimize psychosocial risks and situations of vulnerability in higher education teachers. 


\section{INTRODUÇÃO}

O trabalho desempenha um papel central na vida do ser humano, é fonte de sobrevivência, recompensa profissional e enriquecimento material na medida em que é a partir dele que se retira a remuneração e as recompensas, a sensação de utilidade, valorização pessoal e reconhecimento social. O trabalho adquire uma importância cada vez maior. Por intermédio do trabalho, o indivíduo é inserido numa organização, passa a compartilhar crenças, valores, hábitos, entre outros. Ocupa grande parte do dia a par de aspetos como a família e o lazer, deixa de ser unicamente um meio de sobrevivência ou um ganho económico, para se assumir como um meio de realização. A sua inexistência propícia a criação de um trajeto de fragilização que promove a agudização em escalada de comportamentos como perda de autoestima, dificuldades relacionais, desgaste, frustração, ansiedade, depressão, perturbações do sono, violência, abuso de medicação e de substâncias ilegais (Kilimnik, Neto, Santos, Malta e Santos, 2015; Rodrigues, 1995).

Se, por um lado, o trabalho se assume como um fator de inclusão que previne o aparecimento de situações de risco psicossocial promovendo um estilo de vida saudável por outro lado, há aspetos, dos quais salientamos os relacionados com variáveis de contexto organizacional, que são propiciadores para um possível adoecimento físico e mental.

A União Europeia (European Commission, 2000) destacou como prioritário o estudo dos fatores psicossociais associados ao local de trabalho bem como as consequências que acarretam nos colaboradores, empresas e sociedade (Lluís, Serrano, Corominas, Camps e Giné, 2008).

Por fatores psicossociais entendem-se as características relacionadas com as condições de trabalho que afetam a saúde dos indivíduos, através de processos psicológicos e fisiológicos; estes resultam da interação entre os indivíduos e as condições de vida e trabalho. A literatura disponível permite sustentar a ideia que as condições de trabalho, as novas exigências em torno do exercício profissional e as mudanças nas organizações, em virtude de vários constrangimentos económicos, políticos e sociais, são fatores de risco ao qual não fica isenta a profissão docente como refere Silva et al. (2006).

Borges (2012) defende que nos docentes do ensino superior em Portugal, as mudanças decorrentes do processo de Bolonha, recessão económico-social, diminuição exponencial do número de alunos, sustentabilidade da autonomia das universidades, qualidade do ensino superior são, entre outros, fatores desencadeadores para o aparecimento de situações de mal-estar, insatisfação, stress e desgaste emocional. Este cenário provoca um impacto negativo na qualidade do ambiente psicossocial do trabalho pelo que é imperiosa uma reflexão sobre o atual panorama do ensino superior.

O exercício da profissão docente caracteriza-se por vários seguintes aspetos. Alguns desses aspetos incluem:

"...diversidade das tarefas, interação com os alunos, preparação e implementação de novos métodos de ensino e de tópicos não utilizados anteriormente, oportunidade de organizar o trabalho à sua maneira na sala de aula, imprevisibilidade do quotidiano, processo de encontrar soluções para os problemas, investigação sobre os temas a ensinar, preparação das aulas, novos desafios e o trabalho com os colegas" (Jesus, 2002, p. 223).

Outros aspetos incluem ainda, reconhecimento que alguns alunos e encarregados de educação revelam do trabalho do professor, interesse manifestado pelos alunos durante as aulas, participação em projetos de investigação com os colegas e oportunidades para atualização e aprendizagem através da participação em congressos e ações de formação (Capelo e Calaça, 2016; Costa e Santos, 2013; Ferreira, Machado e Gouveia, 2012; Figueiroa e Moreira, 2014; Formosinho, 2001).

Nas últimas décadas temos assistido por um lado, à desvalorização do papel do docente e, por outro, a um aumento da exigência social sobre esse mesmo papel no processo educativo (Cardoso, Tavares e Sin, 2015; Heitor e Horta, 2015; Jesus et al., 2011). Deste modo, são notórias as referências a respeito do mal-estar símbolo do sinal de declínio na área docente como também, a revelação de que as situações de doença são cada vez mais frequentes e ocorrem com maior intensidade entre a classe docente do que noutros grupos profissionais (Jesus et al., 2011). Reconhece-se que a degradação da condição docente, em termos de remunerações e condições de trabalho em comparação com outras profissões, são fatores que contribuem para um aumento da diminuição do interesse pela profissão, sendo que os professores portugueses apresentam índices de mal-estar superiores aos verificados com professores de outros países europeus, bem como um elevado índice de stress profissional, uma motivação não muito alta e uma maior insatisfação profissional (Jesus et al., 2011; Machado-Taylor et al., 2016).

Esta panorâmica permite-nos compreender o motivo pelo qual o relatório da Organização Internacional do Trabalho (1981) considerou a docência como uma profissão de risco em termos de esgotamento físico e mental dada a exposição exaustiva a condições negativas que promovem o stress (tamanho das turmas, ritmos diferentes de aprendizagem dos alunos, horários pouco adequados, falta de condições de trabalho, 
desvalorização entre os pares e os superiores, pressão para cumprir o programa, ausência de tempo para investir na formação, falta de realização profissional). As condições de exercício da docência estão sujeitas a interferências, como a natureza do trabalho, recompensas pessoais e condições de trabalho de diferente natureza que potenciam desgaste físico e psíquico.

Para Borges (2012) a natureza do trabalho e as recompensas pessoais estão relacionadas respetivamente, com o facto do docente considerar o seu trabalho interessante e significativo pelo que o realiza com satisfação e com aspetos como o salário, progressão na carreira, reconhecimento, relações interpessoais, relação com os colegas e com os órgãos de gestão que influenciam o comprometimento dos docentes. As condições ambientais dizem respeito às condições gerais de conforto físico (temperatura, luz, higiene) que facilitam a execução das funções (condições de segurança, equipamentos de trabalho, localização das instalações). As condições temporais reportam-se à necessidade de delimitar e regular a duração dos períodos de trabalho considerados importantes para a saúde, segurança e preservação da qualidade de vida. As condições de exigência e esforço do trabalho relacionam-se com o esforço físico e/ou mental que a execução da função exige e as condições sociais e organizacionais incluem aspetos como clima organizacional, relações interpessoais, participação nas decisões, circulação da informação, expectativas sociais sobre o trabalho, insuficiência de espaços, desadequação dos equipamentos, inadequação do número de alunos à dimensão da sala, falta de funcionários administrativos, pouco tempo para a investigação, publicação e formação, número elevado de horas de trabalho (tempo de exercício efetivo, tempo de preparação de aulas e correção de testes e exames), horas para reuniões, acompanhamento e atendimento aos alunos que interferem no desempenho docente.

A literatura científica (Bustos, 2005; Correia, 1997; Costa e Santos, 2013; Delgado et al., 1993; Dunham, 2002; Esteve, 1992; Gasparini, Barreto e Assunção, 2005; Gomes, 2014; Jacques, 2003; Kyriacou e Sutcliffe, 1979; Lima e Lima-Filho, 2009; Martinez, 1989; Mercado, 1987 citado em Jesus, 1996; Olivas e Martínez, 2010; Pessoa, 2015; Silva et al., 2016; Vilela, Garcia e Vieira, 2013) revela que as situações de doença ou mal-estar são cada vez mais frequentes e ocorrem com maior intensidade entre a classe docente do que noutros grupos profissionais em termos psicossomáticos, comportamentais, emocionais e motivacionais. A nível psicossomático (fadiga, frustração, perturbações do sono, agressividade, irritabilidade, problemas digestivos e cardiovasculares, ansiedade, perturbações neuróticas e depressivas hipertensão arterial, frequentes dores de cabeça, perda de peso, insónias, úlceras ou desordens intestinais bem como menor resistência às infeções); a nível comportamental (postura conflituosa, abuso de álcool ou drogas, apatia e falta de empenhamento, insegurança); a nível cognitivo (intuição da autoestima e dificuldade na tomada de decisão); a nível emocional (distanciamento afetivo, impaciência, irritabilidade, frustração, apatia) e finalmente, a nível motivacional (perda de idealismo, diminuição do envolvimento e capacidade de iniciativa). Estes sintomas descritos estão associados a situações de trabalho extremamente exigentes no âmbito da profissão docente.

O panorama esboçado interfere na forma como o docente analisa as questões relacionadas com a saúde e a doença, tal como verificou Mendes (1996) ao analisar as representações de um grupo de professores da Universidade de Évora. Foi possível concluir que nos docentes prevalece uma imagem de saúde construída e estruturada a partir da doença e do tempo. A doença delimita todo o processo de valorização da saúde e é o tempo que organiza e orienta todas as decisões que os docentes têm que tomar sobre a saúde e a doença. Num quotidiano preenchido por projetos profissionais, é o tempo de que não dispõem e o tempo que sonham vir um dia a poder dispor, que pressiona a sua agenda. Para os docentes do ensino superior é a espera temporal que medeia todo o processo de tomada de decisões sobre a doença e é o tempo (a falta dele) que os leva a não se preocuparem com a saúde, a não ser quando já não a têm pelo que a sua relação com o tempo é eminentemente social quando se trata de representar a saúde e a doença (Borges, 2012).

Borges (2012) defende que os docentes, com o exercício das múltiplas funções que lhes estão atribuídas, ficam numa situação de vulnerabilidade o que os impede de dar uma adequada e eficaz resposta ao que lhes é solicitado.

Perante o exposto é notório o interesse em abordar os fatores psicossociais que, enquanto características relacionadas com condições e organização do trabalho, interferem na saúde dos trabalhadores, e os riscos psicossociais que surgem da interação entre colaboradores e condições de vida e de trabalho.

Deste modo, o objetivo do estudo é avaliar os fatores de riscos psicossociais em docentes do ensino superior de modo a perceber o exercício da docência enquanto profissão de risco em termos de esgotamento físico e mental.

\section{MÉTODO}

\section{Procedimentos}

Foram cumpridos todos os princípios éticos inerentes a um estudo desta natureza pelo que solicitámos as 
respetivas autorizações aos autores do COPSOQ e ao Conselho Diretivo dos dois estabelecimentos de ensino como também, cumprimos todos os requisitos relacionados com a proteção dos dados.

Neste sentido, os docentes receberam por correio eletrónico informação sobre os objetivos do estudo, indicação de que a participação teria um carácter voluntário e anónimo e que os instrumentos estariam disponíveis online através da aplicação Google Docs de 27/04/2014 a 31/05/2014.

Aplicou-se o teorema do limite central inferindo a normalidade de sua distribuição em amostras com mais de 30 casos, uma vez que este teorema afirma que quando o tamanho de uma amostra aumenta, a distribuição amostral da sua média aproxima-se cada vez mais de uma distribuição normal (Altman e Bland, 1995).

Os dados obtidos foram tratados no programa IBM SPSS Statistics, versão 22, sendo que para os sistematizar utilizámos técnicas de estatística descritiva (média, desvio padrão), correlacional (qui-quadrado entre variáveis qualitativas em que pelo menos uma delas era nominal, e correlação de Spearman quando uma das variáveis em análise era ordinal e a outra quantitativa) e inferencial (teste $t$ de Student e ANOVA para observar diferenças entre dois ou mais grupos respetivamente).

Analisámos ainda a consistência interna do instrumento tendo obtido um alfa de Cronbach de 0,824 o que atesta a consistência do COPSOQ (Peterson, 1994).

O nível do alfa adotado para a significância foi de 0,05.

\section{Amostra}

Pretendeu-se avaliar fatores de risco psicossociais associados ao contexto de trabalho em docentes do ensino superior de dois estabelecimentos de ensino superior de Coimbra: a Escola Superior de Tecnologias da Saúde de Coimbra (ESTeSC) do ensino superior politécnico público e o Instituto Superior Miguel Torga (ISMT) do ensino superior universitário privado.

O estudo realizado foi de cariz descritivo, natureza transversal, tendo sido constituída uma amostra por conveniência e não probabilística.

A população incluiu todos os docentes em exercício de funções no ano letivo de 2013/2014 dos dois estabelecimentos de ensino superior, sendo que foram considerados elegíveis para participar 144 docentes (51 docentes da ESTeSC e 93 do ISMT). Participaram 59 docentes (36 da ESTeSC e 23 do ISMT), o que permite constatar que o nível de adesão foi de 40,9\%.

\section{Instrumentos}

Para a recolha dos dados foram administrados dois instrumentos: um para a caracterização da amostra e outro para avaliar os fatores de risco psicossociais, o Copenhagen Psychosocial Questionnaire (COPSOQ).

O COPSOQ foi desenvolvido e validado, originalmente, por Kristensene Borg (2000), e traduzido e adaptado para a população portuguesa por Silva et al. (2006).

O COPSOQ é um questionário que avalia a exposição do trabalhador a fatores de risco para a saúde, de cariz psicossocial, bem como pretende identificar riscos psicossociais que permitam delinear um plano de prevenção e intervenção em contexto organizacional.

A versão portuguesa está disponível em três versões (curta, média e longa) sendo que a versão média, utilizada no estudo, assume-se como a ferramenta mais adequada não só por apresentar uma identificação mais completa de dimensões psicossociais, mas por ser a versão mais utilizada em estudos internacionais.

Este instrumento, constituído por 76 itens e uma escala de Likert de cinco pontos (1- Nunca/quase nunca, 2- Raramente, 3 - Às vezes, 4 - Frequentemente e 5Sempre), mede indicadores de exposição (riscos psicossociais) e indicadores do seu efeito (saúde, satisfação e stress), tendo em conta cinco dimensões: Exigências Laborais, Organização do Trabalho e Conteúdo, Relações Sociais e de Liderança, Interface Trabalho-Individuo e Valores no Local de Trabalho.

\section{RESULTADOS}

Relativamente à amostra considerou-se as variáveis: sexo, grupo etário, estado civil, filhos e número de filhos, grau académico, categoria profissional, horas lecionadas por semana, vínculo e tempo de serviço na instituição.

$\mathrm{Na}$ Tabela 1 podemos verificar que a distribuição entre o número de homens e mulheres foi quase igual sendo que o sexo masculino foi mais participativo $(50,8 \%)$.

A maioria dos docentes eram casados/união de facto $(69,5 \%)$, com filhos $(64,4 \%)$, na faixa etária entre os 41 e os 50 anos $(45,8 \%)$.

Os docentes possuíam maioritariamente o grau de mestre $(59,3 \%)$ e de doutor $(33,0 \%)$ e $47,5 \%$ eram professores adjuntos e $28,8 \%$ assistentes.

Constatámos que $67,8 \%$ dos docentes tinham uma relação jurídica de emprego estável, fosse em regime de dedicação exclusiva $(35,6 \%)$ fosse a tempo integral $(32,2 \%)$.

Relativamente ao tempo de serviço, 18,7\% dos docentes tinham um vínculo à instituição entre os 14 e os 17 anos e lecionavam entre 14 a 17 horas semanais $(28,8 \%)$. 
Tabela 1

Caraterização da amostra $(N=59)$

\begin{tabular}{|c|c|c|c|}
\hline & & $N$ & $\%$ \\
\hline \multirow{2}{*}{ Sexo } & Feminino & 29 & 49,2 \\
\hline & Masculino & 30 & 50,8 \\
\hline \multirow{3}{*}{ Estado Civil } & Casado(a)/União de Facto & 41 & 69,5 \\
\hline & Separado(a)/Divorciado(a) & 9 & 15,3 \\
\hline & Solteiro & 9 & 15,3 \\
\hline \multirow{3}{*}{ Filhos } & Não & 20 & 33,9 \\
\hline & $\operatorname{Sim}$ & 38 & 64,4 \\
\hline & Não responderam & 1 & 1,7 \\
\hline \multirow{4}{*}{$N^{0}$ de Filhos } & 1 & 15 & 25,4 \\
\hline & 2 & 22 & 37,3 \\
\hline & 3 & 1 & 1,7 \\
\hline & Não responderam & 21 & 35,6 \\
\hline \multirow{5}{*}{ Faixa Etária } & $20-30$ anos & 3 & 5,1 \\
\hline & $31-40$ anos & 21 & 35,6 \\
\hline & $41-50$ anos & 27 & 45,8 \\
\hline & $51-60$ anos & 6 & 10,2 \\
\hline & +60 anos & 2 & 3,4 \\
\hline \multirow{3}{*}{ Grau Académico } & Mestrado & 35 & 59,3 \\
\hline & Doutoramento & 23 & 39,0 \\
\hline & Pós-Doutoramento & 1 & 1,7 \\
\hline \multirow{5}{*}{ Categoria Profissional } & Assistente & 17 & 28,8 \\
\hline & Prof. Adjunto & 28 & 47,5 \\
\hline & Prof. Auxiliar & 10 & 16,9 \\
\hline & Prof. Coordenador & 2 & 3,4 \\
\hline & Prof. Associado & 2 & 3,4 \\
\hline \multirow{4}{*}{ Contrato de Trabalho } & Prestação serviços (mês/hora) & 13 & 22,0 \\
\hline & Tempo integral & 19 & 32,2 \\
\hline & Tempo parcial & 6 & 10,2 \\
\hline & Dedicação exclusiva & 21 & 35,6 \\
\hline \multirow{8}{*}{ Anos de Serviço } & Entre 1 e 3 anos & 7 & 11,9 \\
\hline & Entre 4 e 6 anos & 10 & 12,1 \\
\hline & Entre 7 e 10 anos & 9 & 15,3 \\
\hline & Entre 11 e 13 anos & 9 & 15,3 \\
\hline & Entre 14 e 17 anos & 11 & 18,7 \\
\hline & Entre 18 e 21 anos & 6 & 10,2 \\
\hline & Mais de 22 anos & 3 & 5,1 \\
\hline & Não responderam & 1 & 1,7 \\
\hline \multirow{6}{*}{$N^{0}$ de aulas lecionadas } & Entre 1 e 3 horas & 1 & 1,7 \\
\hline & Entre 4 e 6 horas & 9 & 15,3 \\
\hline & Entre 7 e 10 horas & 10 & 17,0 \\
\hline & Entre 11 e 13 horas & 21 & 35,6 \\
\hline & Entre 14 e 17 horas & 17 & 28,8 \\
\hline & Mais de 18 horas & 1 & 1,7 \\
\hline Total & & 59 & 100,0 \\
\hline
\end{tabular}

Quando se avaliaram os fatores do risco psicossociais (Gráfico 1) constatou-se como mais relevantes os relacionados com a dimensão Exigências Laborais: as subescalas Exigências Cognitivas e Exigências Emocionais foram as que apresentam valores mais altos, isto é, potenciavam uma exposição mais desfavorável para a saúde dos docentes em termos de riscos psicossociais. $\mathrm{Na}$ dimensão Organização do Trabalho e Conteúdo, as subescalas: Possibilidade de Desenvolvimento e Significado do Trabalho assumiram uma situação de risco mais desfavorável para a saúde dos docentes. No que diz respeito às Relações Sociais e Liderança, destacamos a Transparência do Papel Laboral Desempenhado; esta dimensão revelou-se como de maior risco para os docentes em termos psicossociais. Finalmente, na dimensão Interface-Indivíduo, a subescala Conflito Trabalho/Família e Insegurança Laboral revelou maior risco para a saúde.

A nível da situação intermédia que nos indica a vulnerabilidade face ao risco, destacamos na dimensão Exigências Laborais, as Exigências Quantitativas; na Organização do Trabalho e Conteúdo a subescala Influência no Trabalho; nas Relações Sociais e Liderança destacamos a subescala Apoio Social dos Superiores, Conflitos de Papéis Laborais, Previsibilidade e Apoio Social dos Colegas.

Na dimensão Interface Trabalho-Indivíduo destacouse a subescala Satisfação Laboral e na dimensão Valores no Local de Trabalho a subescala Confiança Horizontal que apresentou valores mais elevados ao nível da situação intermédia.

Comparámos os dados a nível das subescalas com o tipo de estabelecimento de ensino, tendo-se recorrido ao teste $t$ de Student. Os valores apresentados pelas duas instituições de ensino superior mostraram não existir diferenças significativas a assinalar $(p>0,05)$. No entanto, obtivemos resultados estatisticamente significativos $(p<0,05)$ nas subescalas Problemas em Dormir e Comportamentos Ofensivos que foram mais elevados na instituição de ensino pública do que na privada (respetivamente, $M=2,73 ; D P=1,22$ e $M$ $=1,23 ; D P=0,43$.

Quanto se comparam os valores da média obtida no estudo com os valores de referência da amostra global e a da área do ensino verificou-se que os dados foram muito idênticos.

Destacamos as subescalas Justiça e Respeito (instituição pública: $M=2,93 ; D P=0,94$; instituição privada: $M=2,84 ; D P=0,70 ; t=0,65 ; p=0,516) \mathrm{e}$ Significado de Trabalho (instituição pública: $M=4,31 ; D P$ $=0,69$; instituição privada: $M=4,39 ; D P=0,64 ; t=0,70$; $p=0,486$ ) que apresentaram valores mais baixos e mais altos comparativamente aos valores de referência global (Justiça e Respeito: $M=4,34 ; D P=0,79 /$ Significado do Trabalho: $M=3,77 ; D P=0,67$ ) e de ensino (Justiça e Respeito: $M=3,98 ; D P=0,67$; Significado do Trabalho: $M=3,52 ; D P=0,89$ ).

Quanto ao sexo, através do qui-quadrado verificámos a existência de diferenças significativas $(p \leq$ 0,05) com o Ritmo de Trabalho e Burnout, o que constituiu para o sexo feminino um risco para a saúde enquanto no masculino a Previsibilidade e Recompensas foram consideradas duas situações de risco psicossocial. 


\section{Gráfico 1}

\section{Risco Psicossocial dos Docentes}

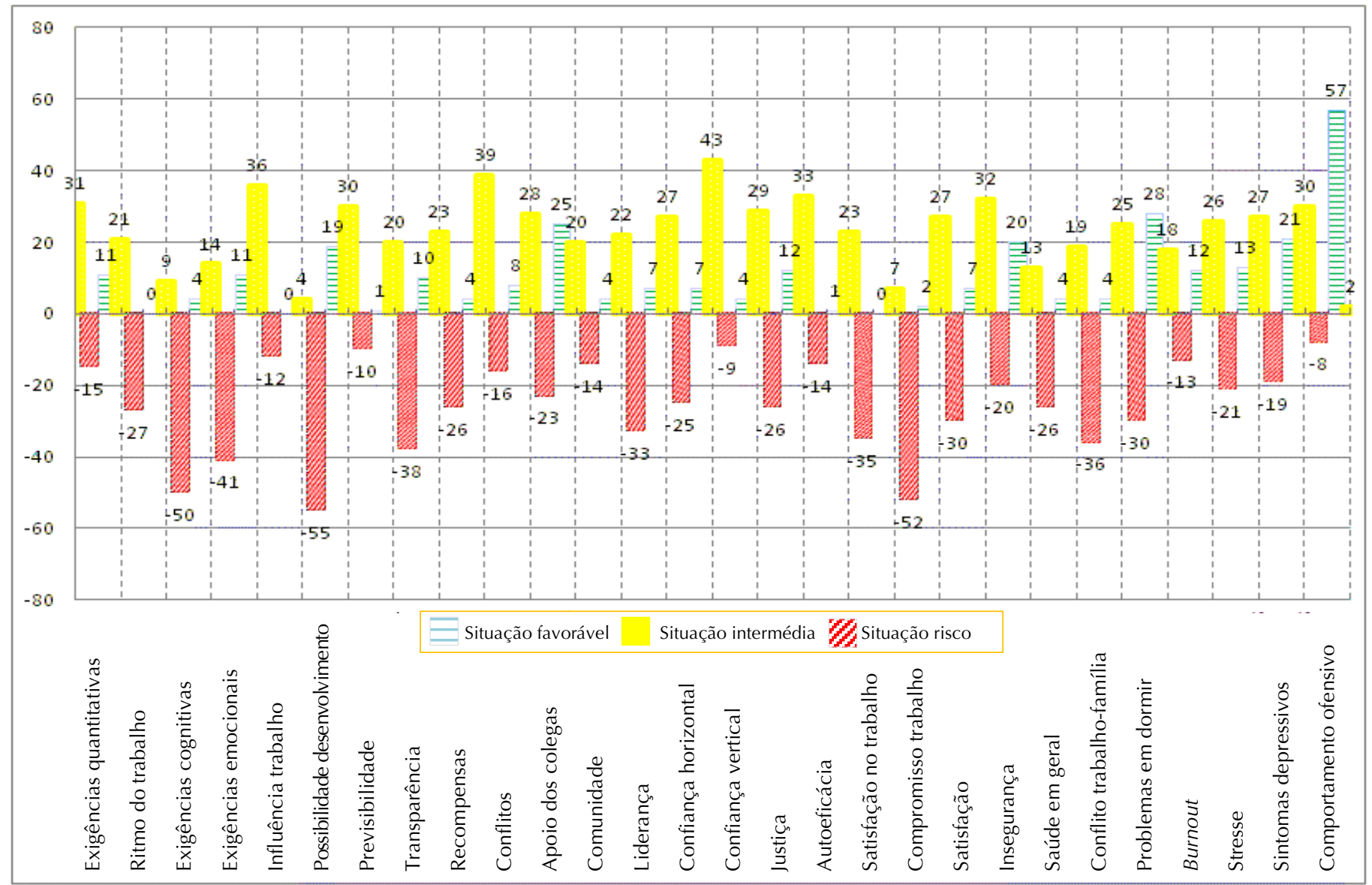

Quanto à variável idade, procedemos à correlação (coeficiente de correlação de Spearman) e na subescala Confiança Horizontal, obtivemos uma relação estatisticamente significativa $(\rho=0,288$; $p<$ $0,05)$. As subescalas Possibilidade de Desenvolvimento $(\rho=-0,278 ; p<0,05)$, Compromisso com o Trabalho ( $\rho$ $=-0,277 ; p<0,05)$ e Confiança Horizontal $(\rho=-0,301$; $p<0,05)$ revelaram-se uma situação de risco para a saúde com o aumento da idade dos docentes.

No que reporta à formação académica quando correlacionada com as subescalas, o Apoio Social de Colegas $(\rho=-0,261 ; p<0,05)$ destacou-se como uma situação desfavorável para a saúde dos docentes com grau académico mais elevado.

Encontraram-se diferenças estatisticamente significativas $(p<0,05)$ entre os Riscos para a saúde e a categoria profissional, sendo os professores coordenadores os que apresentaram maiores riscos para a saúde $(M=5,00 ; D P=1,00)$ e a Saúde dos professores associados foi aquela que apresentou menor risco $(M=$ 2,00; $D P=1,41)$. A Qualidade da Liderança apresentou valores compatíveis com risco estatisticamente significativo $(p<0,05)$ para os assistentes $(M=3,75$; $D P$
$=0,54)$ e para os professores associados $(M=3,88 ; D P=$ $0,88)$.

A correlação de Spearman entre o número de horas lecionadas e a subescala Comunidade Social no Trabalho, revelou uma correlação fraca $(\rho=-0,308)$ contudo estatisticamente significativa $(p<0,05)$.

A correlação entre o tempo de serviço e a subescala Exigências Quantitativas foi igualmente fraca $(0,288)$, mas significativa $(p<0,05)$.

\section{DISCUSSÃO}

Os riscos psicossociais associados ao exercício profissional são compostos por um conjunto de fatores que comprometem o bem-estar físico e mental dos profissionais em contexto de trabalho independentemente, do sexo ou idade dos docentes.

$\mathrm{O}$ estudo realizado permite verificar que participaram mais docentes do sexo masculino, com idade entre os 41 e os 50 anos, o que vai ao encontro dos dados obtidos por Schluter e Valdéz (2001), em que participaram 60 professores universitários (15 eram do sexo masculino e 15 do feminino de uma universidade privada e o mesmo 
número de uma universidade pública), e por Marqueze e Moreno (2009), na Universidade do Extremo Sul Catarinense, em que dos 154 docentes 50,6\% eram do sexo masculino, com idade média de 39,25 anos. Já o estudo de Carlotto e Câmara (2004) com uma amostra de 280 professores universitários de uma Universidade de Porto Alegre, de Araújo, Sena, Viana e Araújo (2005) na Universidade Estadual de Feira de Santana (UEFS), de Borges (2012) com uma amostra de 81 docentes do ensino superior português, de Pessoa (2015) com 100 docentes brasileiros de uma cidade do Estado do Maranhão e de Bueno (2017) com 26 professores brasileiros das Escolas Pantaneiras, a população mais participativa foi a do sexo feminino.

A literatura científica demonstra as consequências dos riscos psicossociais para as organizações, o que tem implicações ao nível da saúde pública, saúde física e mental dos docentes do ensino superior.

Sendo a docência considerada como uma das atividades que apresenta um leque de conteúdos cognitivos, efetivos e instrumentais, que interferem na qualidade de vida/saúde, assume-se como uma atividade profissional desgastante devido às caraterísticas do trabalho e do papel de docente, conflitos interpessoais para além da baixa remuneração, estagnação na carreira e inadequação da estrutura dos estabelecimentos de ensino, o que contribui para o aparecimento de cada vez mais situações de risco psicossocial ao nível na profissão docente.

O estudo de Pessoa (2015) destaca os vários riscos psicossociais que interferem de modo negativo na capacidade de trabalho dos docentes como stress, apoio social de superiores, insegurança laboral, satisfação no trabalho e transparência no papel laboral. A autora considera que riscos psicossociais, como a pressão no ambiente de trabalho, excessiva carga de trabalho física ou mental e a continuidade das tarefas de trabalho que muitas vezes se estendem ao lar e interferem nas relações familiares, influenciam a saúde mental dos docentes e são propiciadoras de desequilíbrios.

Bueno (2017) apresenta como principais riscos psicossociais para a saúde dos professores as exigências quantitativas, ritmo de trabalho, falta de influência no trabalho, problemas relacionados ao sono, extenuação, stress psíquico, somático e cognitivo, sintomas depressivos e problemas no relacionamento interpessoal.

A degradação da condição docente em termos de remunerações e condições de trabalho, em comparação com outras profissões, propiciam diminuição do interesse pela profissão, baixa motivação e uma menor satisfação profissional, pelo que Borges (2012) no seu estudo desenha um perfil de docentes que trabalha no "fio da navalha" a programar, preparar aulas, avaliar, orientar, atender alunos, desempenhar tarefas administrativas, assistir e participar em seminários e reuniões de índole diversa para além da investigação, publicação e preocupações com prosseguimento na carreira.

Para Mendes (1996), Franco (2001) e Minter (2009), os docentes do ensino superior tem múltiplas missões que vão desde o ensino e serviço à comunidade, à investigação e criação de conhecimento. No trabalho docente há necessidade contínua de estudo e aperfeiçoamento, como também, o trabalho não termina no fim do expediente.

De igual modo, o relevo que se dá à produção científica em detrimento do ensino afeta a imagem que o docente tem de si, dado que as suas eventuais promoções nunca dependerão das suas qualidades pedagógicas, mas do destaque obtido com trabalhos de pesquisa científica, o que como refere Mendes (1996, p. 168) "subverte a vertente dominante da sua carreira - o ensino".

A cada vez maior visibilidade deste paradigma interfere com aspetos como a Satisfação no Trabalho, Justiça e Respeito, mas também com as questões da Previsibilidade, Insegurança Laboral e Compromisso face ao Local de Trabalho, o que vai corroborar os dados obtidos neste estudo e na revisão da literatura (Álvarez Flores, 2007; Carlotto e Câmara, 2004, 2017; Costa e Santos, 2013; Dipp, Flores e Gutiérrez, 2010; Gomes, 2014; Marqueze e Moreno, 2009; Pessoa, 2015; Sousa e Mendonça, 2009; Vilela, Garcia e Vieira, 2013).

No que diz respeito à Satisfação no Trabalho, esta relaciona-se com a perceção que os docentes têm sobre as condições de exercício profissional e a utilização das suas capacidades no desempenho. A Justiça e Respeito demonstram a influência que os valores, atitudes e sentimentos têm sobre o que é justo ou injusto. Sendo os docentes uma classe profissional sensível à justiça, é previsível que atuem em conformidade nos processos de avaliação de desempenho, decisões de promoção, incrementos salariais e controlo das atividades, uma vez que os docentes não têm idêntica configuração na sua atividade porque reportam a vários superiores no plano administrativo, científico e pedagógico.

No meio docente constata-se que a entidade que distribui as recompensas é a mesma que distribui as tarefas, pelo que os professores a distinguem; porém, a entidade que distribui o serviço docente não tem correspondente controlo sobre as recompensas.

Como refere Borges (2012, p. 50), “A distribuição do serviço docente é feita semestre a semestre ou ano a ano, havendo muitas vezes flutuações no modo como é desenhada periodicamente, o que não tem correspondência exata no plano das remunerações. Para 
além do desempenho de atividades advindas da distribuição do serviço docente, os professores executam diversas funções cuja quantidade, modo de exercício e desempenho não têm implicações na remuneração".

A Previsibilidade relaciona-se com a transmissão da informação adequada e em tempo suficiente para os docentes se adaptarem às mudanças, o que poderá provocar o aumento dos níveis de stress. Em relação à situação laboral, é importante que sejam comunicadas em tempo útil aos docentes informação sobre o serviço atribuído e sobre eventuais reestruturações e mudanças organizacionais. Neste aspeto, a comunicação interna é crucial dado que permite o ajustamento de falhas, proporciona oportunidades para o desenvolvimento da aprendizagem e habilidades, bem como permite envolver os docentes na estratégia de desenvolvimento da organização (Huse, 1989 citado por Ferreira, Neves e Caetano, 2001; Kanter, Stein e Jick, 1992 citado por Ferreira e Martinez, 2008; Lawler, 1986 citado por Ferreira e Martinez, 2008). Decorrente da situação anterior, surge a questão da Insegurança Laboral, que tanto pode ser contratual como sobre as condições de trabalho (mobilidade funcional e geográfica, mudanças do horário de trabalho e do salário) ou sobre ajustamentos organizacionais, o que representa um risco para a saúde.

No que se refere ao Compromisso face ao Local de Trabalho, esta é uma situação favorável para a saúde que aumenta com a idade (falar com os outros sobre o local trabalho, sentir os problemas do local de trabalho como seus); associado a esta questão está a manutenção do ambiente de trabalho, em termos psicossociais, dado que melhora a saúde e o bem-estar dos trabalhadores, fomenta a produtividade, estimula o desenvolvimento profissional bem como o desempenho económico, promove a abstenção e a rotatividade de mão-de-obra.

Associado à manifestação de riscos psicossociais, o Burnout potencia a acumulação de fadiga (física e mental) e a exaustão mental e destaca-se no sexo feminino. A acumulação de atividades docentes e a sua conciliação com o exercício de diferentes papéis sociais tem implicações no ritmo de trabalho das mulheres docentes. O Ritmo de Trabalho acelerado ocasiona a falta de tempo, não só para as suas atividades diárias como para pensar em si, pelo que a negação da doença é um facto dado que serve para não recorrerem ao médico, como também para adiar o conhecimento sobre o problema que pode estar a perturbar o ritmo de trabalho que querem assegurar como afirma Mendes (1996).

Carlotto e Câmara $(2004,2017)$ e Carlotto e Palazzo (2006) estudaram a síndrome de Burnout em professores universitários e encontrou uma associação negativa entre as dimensões de Burnout e Características de cargo e, no que respeita à exaustão emocional, evidenciou associação com identificação com a tarefa, autonomia e potencial motivacional do cargo.

Marqueze e Moreno (2009) verificaram que há aspetos com os quais há maior proporção de docentes no nível de satisfação, como o conteúdo do trabalho que realiza, relacionamento com outras pessoas na instituição e grau de motivação para o trabalho e, por outro, há aspetos com maior proporção de docentes no nível de insatisfação, como o volume de trabalho que tem para desenvolver, grau de segurança (estabilidade) no emprego e grau em que a instituição absorve as potencialidades que julga ter.

Quanto aos dados que obtivemos, estes apresentam risco psicossocial nas subescalas Exigências Cognitivas, Possibilidade de Desenvolvimento e Significado do Trabalho e nas subescalas Confiança Horizontal, Conflito, Influência no Trabalho e Exigências Quantitativas, indicando uma situação de vulnerabilidade dos docentes face à situação de risco.

O estudo de Pessoa (2015) revela que as variáveis com valores mais elevados e significativos foram a influência no trabalho e o conflito laboral. Verifica-se, ainda, de forma negativa a variável Comportamentos Ofensivos sendo que o estudo sugere que o sexo masculino influencia mais negativamente, em comparação com o feminino, os Comportamentos Ofensivos.

Salientamos as subescalas Justiça e Respeito e Significado do Trabalho bem como Ritmo de Trabalho, Previsibilidade, Recompensas, Possibilidade de Desenvolvimento e Insegurança Laboral em que foram encontrados dados que despertaram interesse para futuros estudos nomeadamente, no que concerne ao sexo, idade, formação académica e categoria profissional. Os dados obtidos vão ao encontro dos estudos realizados por Bueno (2017), Costa e Santos (2013) e Pessoa (2015).

Neste sentido, Gago e Correia (2010), ao analisarem 84 professores de vários níveis de ensino, demostraram que perante a injustiça procedimental os participantes com maior crença pessoal no mundo justo reagem mais positivamente (com mais paciência) comparativamente com os que têm baixa crença pessoal no mundo justo. No entanto, os participantes com alta crença pessoal no mundo justo reagiram à injustiça procedimental mais negativamente (negligência e voz agressiva) comparativamente com aqueles que tinham baixa crença pessoal no mundo justo.

Sousa e Mendonça (2009) na pesquisa com 233 professores universitários verificaram que o poder mediacional do comprometimento se confirmou na relação entre perceção de justiça distributiva e exaustão, pelo que a perceção de injustiça na forma de distribuição de recursos pode levar o professor universitário à 
exaustão, o que pode ter probabilidade aumentada diante da falta de comprometimento.

Finalmente, destacamos os valores obtidos ao nível das subescalas Possibilidade de Desenvolvimento, Significado do Trabalho e Exigências Cognitivas, que vão ao encontro do atual panorama profissional dos docentes do ensino superior. As referidas escalas são percecionadas como situações de risco para a saúde no que se refere à Possibilidade de Desenvolvimento que a carreira proporciona.

O trabalho docente é uma fonte de oportunidades, de desenvolvimento de competências e conhecimento. Pode ser criativo e exercido com autonomia, no entanto, quando começa a ser percecionado como monótono e repetitivo, não representa aprendizagem, crescimento ou possibilidade de desenvolvimento pelo que potencia a manifestação de situações de risco psicossocial.

Por um lado, as exigências de desenvolvimento ao nível da obtenção do grau de Doutor e de produção científica sem implicações de promoção na carreira ou remuneratórias, traz consequências negativas e, por outro, as mesmas exigências permitem ascender na carreira em termos científicos. Esta dualidade proporciona e legítima os docentes para desenvolver atividades de gestão, coordenação e direção, participação nos órgãos de decisão científica e pedagógica, integração em provas académicas e participação ativa em projetos de pesquisa internos e externos à instituição, mas descura a progressão na carreira e o patamar salarial.

Quanto mais elevada for a categoria profissional dos docentes, mais sobrecarregados estão de trabalho e mais dificuldades sentem em dar uma resposta adequada e eficaz a todas as solicitações. Apesar de reconhecermos que há atrativos na docência, nomeadamente, períodos de férias dos alunos, grau de autonomia e flexibilidade horária a agenda do docente é preenchida por atividades de diferente natureza que provocam sobrecarga e dificuldades em geri-la de modo equilibrado.

Assim, o docente está exposto a condições de trabalho (insuficiência de espaços, desadequação dos equipamentos, inadequação do número de alunos à dimensão da sala, falta de funcionários administrativos, pouco tempo para a investigação, publicação e formação, número elevado de horas de trabalho, tempo de exercício efetivo, tempo de preparação de aulas e correção de testes e exames, reuniões, acompanhamento e atendimento aos alunos) que colocam em risco a saúde e impedem a sua realização profissional.

O presente estudo, apesar de ter abordado de forma exploratória a relação que algumas variáveis têm com as várias subescalas do COPSOQ e avaliado os fatores de risco psicossociais mais significativos que expõem os docentes a uma maior situação de vulnerabilidade, apresenta limitações metodológicas porque se centra sobre uma população constituída por docentes de dois estabelecimentos de ensino superior onde os autores do estudo lecionam.

A este facto consideramos que os docentes do ensino superior assoberbados com a realização de inúmeras funções ficam sem tempo e espaço para repensar-se enquanto classe profissional. Em futuros estudos, será conveniente alargar a recolha de dados a docentes de outros estabelecimentos de ensino superior, considerar de forma representativa os diversos sistemas de ensino superior em vigor em Portugal bem como alargar o estudo a estabelecimentos de ensino de outros países.

É importante destacar que as boas condições psicossociais em contexto laboral contribuem para a qualidade de vida dos profissionais, o que resultará no aumento da sua capacidade para o trabalho. Deste modo, preconizamos a realização de estudos que permitam melhor compreender a temática em análise dando seguimento aos estudos de Borges (2012), Bueno (2017), Capelo e Calaça (2016), Cortez Souza, Amaral e Silva (2017), Figueiroa e Moreira (2014), Fragoeiro (2011), Pessoa (2015), Sousa et al. (2017), bem como aos realizados pela Agência Europeia para a Segurança e Saúde no Trabalho (2018), seja ao nível da prevalência de riscos psicossociais e estratégias de prevenção, seja ao nível da prevenção do stress relacionado com o trabalho no setor educacional.

Em face das evidências de situações de risco para a saúde nos docentes é imperativa a avaliação e gestão dos riscos psicossociais com vista à sua proteção, de forma a promover condições de trabalho saudáveis, estimular a clareza e transparência organizativa, garantir respeito e tratamento justo, bem como incentivar a promoção da conciliação vida profissional/familiar, de modo a minimizar riscos psicossociais e situações de vulnerabilidade nos docentes do ensino superior.

Os autores têm intenção de dar continuidade ao estudo, dado que se constata uma enorme lacuna de estudos ao nível dos docentes do ensino superior bem como pretendem comunicá-los aos órgãos próprios de ambas as instituições de forma a poder iniciar-se uma estratégia de mudança fundamental com a elaboração de estratégias de promoção da saúde e uma gestão preventiva do risco, através da introdução de atividades planeadas e sistemáticas, de modo, a assegurar a sua eficácia, de modo a obter resultados benéficos ao nível do estado de saúde e do desempenho.

O objetivo é promover condições de vida no trabalho a nível individual ou organizacional nomeadamente, ao nível da intervenção primária (reestruturação de medidas organizacionais, mudanças no processo de tomada de 
decisão, enriquecimento funcional, reorganização das linhas de autoridade, redesenho do layout físico, estabelecimento de um sistema de compensação mais justo); intervenção secundária (treino de relaxamento, desenvolvimento de capacidades de gestão de tempo ou de resolução de conflitos) e, finalmente, ao nível da intervenção terciária (abordagens que envolvem o aconselhamento individual, tendo em conta a identificação tipo de risco, e também o diagnóstico de possíveis efeitos negativos noutras esferas da vida do docente).

\section{CONCLUSÃO}

Em conclusão, o presente estudo indica uma situação de vulnerabilidade dos docentes do ensino superior face à situação de risco psicossocial nas subescalas Exigências Cognitivas, Possibilidade de Desenvolvimento e Significado do Trabalho e nas subescalas Confiança Horizontal, Conflito, Influência no Trabalho e Exigências Quantitativas.

Os valores apresentados pelas duas instituições de ensino superior mostraram não existir diferenças significativas a assinalar, no entanto, obtivemos resultados estatisticamente significativos na subescala Problemas em Dormir e Comportamentos Ofensivos que foram mais elevados na instituição de ensino pública do que na privada.

Verifica-se, ainda, de forma negativa a variável Comportamentos Ofensivos, sendo que o estudo sugere que o sexo masculino influencia mais negativamente, em comparação com o feminino, os referidos comportamentos.

Salientamos as subescalas Justiça e Respeito e Significado do Trabalho, Ritmo de Trabalho, Previsibilidade, Recompensas, Possibilidade de Desenvolvimento e Insegurança Laboral em que foram encontrados dados que despertaram interesse, nomeadamente, no que concerne ao sexo, idade, formação e grau académico e categoria profissional.

Os presentes resultados podem ter implicações a nível prático na promoção de condições de vida no trabalho a nível individual e organizacional, seja a nível da intervenção primária e secundária, seja a nível da intervenção terciária. Perante as evidências de situações de risco para a saúde nos docentes do ensino superior urge avaliar os riscos psicossociais, promover condições de trabalho saudáveis, estimular a clareza e transparência organizativa, garantir respeito e tratamento justo e incentivar a promoção da conciliação vida profissional/familiar de modo a minimizar riscos e situações de vulnerabilidade psicossociais.
Conflito de interesses | Conflict of interest: nenhum | none.

Fontes de financiamento | Funding sources: nenhuma | none.

\section{REFERÊNCIAS}

Agência Europeia para a Segurança e Saúde no Trabalho. (2018). Os riscos psicossociais na Europa: Prevalência e estratégias de prevenção Recuperado de https://osha.europa.eu/pt/tools-andpublications/publications/reports/executive-summary-psychosocialrisks-in-europe-prevalence-and-strategies-for-prevention/view

Altman, D. G. e Bland, J. M. (1995). Statistics notes: The normal distribution. BMJ, 310(6975), 298. doi:10.1136/bmj.310.6975.298

Álvarez Flores, D. (2007). Satisfacción y fuentes de presión laboral en docentes universitarios de Lima metropolitana. Persona, (10), 49-97. Recuperado de http://www.redalyc.org/articulo.oa?id=147112813004

Araújo, T. M., Sena, I. P., Viana, M. A. e Araújo, E. M. (2005). Mal-estar docente: Avaliação de condições de trabalho e saúde em uma instituição de ensino superior. Revista Baiana de Saúde Pública, 29(1), 6-21. Recuperado de http://pesquisa.bvsalud.org/sms/resource/pt/lil-416272

Borges, S. C. M. L. (2012). Satisfacción en el trabajo y salud mental en docentes de ensenanza superior de Coimbra (Tese de Doutoramento, Universidad de Extremadura, Badajoz). Disponível em http://hdl.handle.net/10662/316

Bueno, H. P. V. (2017). Fatores de riscos psicossociais em professores de escolas pantaneiras: Relações com transtornos mentais comuns e estresse ocupacional. (Tese de Doutoramento, Universidade Católica Dom Bosco, Campo Grande). Recuperado de http://site.ucdb.br/public/md-dissertacoes/1018294-helen-final.pdf

Bustos, M. I. C. (2005). La realidad escolar cotidiana y la salud mental de los profesores. Revista Enfoques Educacionales, 7(1), 69-79. Recuperado de http://www.facso.uchile.cl/publicaciones/enfoques/09/Corvalan_N7_20 05.pdf

Capelo, M. R. T. F. e Calaça, Z. V. A. (2016). O poder preditivo do coping e do engagement no stresse laboral dos professores do ensino especial. Informes Psicológicos, 16(2), 13-25. doi:10.18566/infpsicv16n2a01

Cardoso, S., Tavares, O. e Sin, C. (2015). The quality of teaching staff: Higher education institutions' compliance with the European Standards and Guidelines for Quality Assurance - the case of Portugal. Educational Assessment, Evaluation and Accountability, 27(3), 205-222. doi:10.1007/s11092-015-9211-z

Carlotto, M. S. e Câmara, S. G. (2004). Análise fatorial do Maslach Burnout Inventory $(\mathrm{MBI})$ em uma amostra de professores de instituições particulares. Psicologia em Estudo, 9(3), 499-505. doi:10.1590/S1413-73722004000300018

Carlotto, M. S. e Câmara, S. G. (2017). Riscos psicossociais associados à síndrome de burnout em professores universitários. Avances en Psicología Latinoamericana, 35(3), 447-457. doi:10.12804/revistas.urosario.edu.co/apl/a.4036

Carlotto, M. S. e Palazzo, L. S. (2006). Síndrome de burnout e fatores associados: Um estudo epidemiológico com professores. Cadernos de Saúde Pública, 22(5), 1017-1026. doi:10.1590/S0102$311 \times 2006000500014$

Correia, V. (1997). Estudo do bem-estar e mal-estar na profissão docente em Educação Física: O stress profissional (Dissertação de mestrado não publicada). Universidade Técnica de Lisboa, Lisboa.

Cortez, P. A., Souza, M. V. R., Amaral, L. O. e Silva, L. C. A. (2017). A saúde docente no trabalho: Apontamentos a partir da literatura recente. Cadernos Saúde Coletiva, 25(1), 113-122. doi:10.1590/1414-462×201700010001

Costa, L. S. e Santos, M. (2013). Fatores psicossociais de risco no trabalho: Lições aprendidas e novos caminhos. International Journal on Working Conditions, 1(5), 39-58. Recuperado de http://ricot.com.pt/artigos/1/IJWC.5_LSCosta.MSantos_39.58.pdf

Delgado, A. C., Fuentes, J. M. B., Quevedo, M. P. A., Salgado, A. R., Sánchez, A. C., Sanchez, T. S., ... Yela, J. R. B. (1993). Revisión teórica del burnout o desgaste profesional en trabajadores de la docencia. Caesura, (2), 47-65. 
Dipp, A. J., Flores, J. A. T. e Gutiérrez, R. V. (2010). Satisfacción laboral y compromiso institucional de los docentes de posgrado. Revista Eletrónica Diálogos Educativos, 10(19), 119-130. Recuperado de https://dialnet.unirioja.es/servlet/articulo?codigo=3294739

Dunham, J. (2002). Stress in teaching (2. ${ }^{\mathrm{a}}$ ed.). London, UK: Routledge.

Esteve, J. M. (1992). O mal-estar docente. Lisboa: Escher/ Fim de Século Edições.

European Commission. (2000). Guidance on work-related stress: Spice of like or kiss of death? Luxembourg: Office for Official Publications of the European Communities. Recuperado de https://www.stress.org/wpcontent/uploads/2011/11/Guidance2520on2520workrealted2520stress.pdf

Ferreira, A. I. e Martinez, L. F. (2008). Manual de diagnóstico e mudança organizacional. Lisboa: RH Editora.

Ferreira, J. B., Machado, M. L. e Gouveia, O. (2012). A (in)satisfação dos académicos no ensino superior. Educação, Sociedade e Culturas, (37), 129-139.

Ferreira, J. M. C., Neves, J. G. e Caetano, A. (2001). Manual de psicossociologia das organizações. Lisboa: McGraw-Hill.

Figueiroa, A. e Moreira, A. (2014). Initial teacher training: Contribution to an appropriate use of the experimental work. American Journal of Educational Research, 2(8), 629-634. doi:10.12691/education-2-8-12

Formosinho, J. (2001). A formação prática de professores: Da prática docente na instituição de formação à prática pedagógica nas escolas. Em B. P. Campos, (Org.), Formação profissional dos professores no ensino superior (pp. 46-64). Porto: Porto Editora.

Fragoeiro, J. R. A. G. (2011). Satisfação dos docentes do ensino superior. (Dissertação de mestrado, Universidade da Madeira, Madeira). Disponível em http://hdl.handle.net/10400.13/445

Franco, M. E. D. P. (2001). Comunidade de conhecimento, pesquisa e formação do professor do ensino superior. Em M. C. Morosini (Org.) Professor do ensino superior: Identidade, docência e formação (pp. 61-73). Brasília: Plano Editora.

Gago, A. R. e Correia I. (2010). Reacções à injustiça no trabalho: Impacto da crença no mundo justo, da justiça procedimental e da justiça distributiva. Análise Psicológica, 28(1), 59-70. Recuperado de http://www.scielo.oces.mctes.pt/pdf/aps/v28n1/v28n1a05.pdf

Gasparini, S. M., Barreto, S. M. e Assunção, A. A. (2005). O professor, as condições de trabalho e os efeitos sobre sua saúde. Educação e Pesquisa, 31(2), 189-199. doi:10.1590/S151797022005000200003

Gomes, A. F. P. (2014). O impacto dos fatores psicossociais na saúde mental (Dissertação de Mestrado, Universidade de Aveiro, Aveiro). Disponível em http://hdl.handle.net/10773/12696

Heitor, M. e Horta, H. (2015). Reforming higher education in Portugal in times of uncertainty: The importance of illities, as non-functional requirements. Technological Forecasting and Social Change, 113(B), 146-156. doi:10.1016/j.techfore.2015.09.027

Jacques, M. G. C. (2003). Abordagens teórico-metodológicas em saúde/doença mental e trabalho. Psicologia \& Sociedade, 15(1), 97116. doi:10.1590/S0102-71822003000100006

Jesus, S. N. (1996). A motivação para a profissão docente: Contributo para a clarificação de situações de mal-estar e para a fundamentação de estratégias de formação de professores (Tese de Doutoramento, Universidade de Coimbra, Coimbra). Disponível em http://hdl.handle.net/10316/1042

Jesus, S. N. (2002). Perspetivas para o bem-estar docente - Uma lição de síntese. Porto: Edições ASA.

Jesus, S. N., Mosquera, J., Stobaus, C., Sampaio, A., Rezende, M. e Mascarenhas, S. (2011). Avaliação da motivação e do bem/mal-estar dos professores: Estudo comparativo entre Portugal e Brasil. Revista AMAzônica, $\quad 7(2), \quad 7-18 . \quad$ Disponível em http://hdl.handle.net/10923/8632

Kilimnik, Z. M., Neto, M. T. R., Santos, G. S., Malta, V. D. e Santos, M. F. (2015). O significado do trabalho: Um estudo com professores de administração em uma universidade. Revista Lugares de Educação, 5(11), 3-27. doi:10.18788/2237-1451/rle.v5n11p3-27
Kristensen, T. S. e Borg, V. (2000). Copenhagen Psychosocial Questionnaire (COPSOQ). Copenhagen: National Institute of Occupational Health.

Kyriacou, C. e Sutcliffe, J. (1979). Teacher stress and satisfaction. Educational Research, 21(2), 89-96. doi:10.1080/0013188790210202

Lima, M. F. E. M. e Lima-Filho, D. O. (2009). Condições de trabalho e saúde do/a professor/a universitário/a. Ciências \& Cognição, 14(3), 62-82. Recuperado http://www.cienciasecognicao.org/pdf/v14_3/m253.pdf

Lluís, S. M., Serrano, C. L., Corominas, A. F., Camps, A. G. e Giné, A. N. (2008). Exposición a riesgos psicosociales entre la población asalariada en España (2004-05): Valores de referencia de las 21 dimensiones del cuestionario COPSOQ ISTAS21. Revista Espanõla de Salud Pública, 82(6), 667-675. doi:10.1590/S113557272008000600007

Machado-Taylor, M. L., Meira Soares, V., Brites, R., Brites Ferreira, J., Farhangmehr, M., Rocha Gouveia, O. M. e Peterson, M. (2016). Academic job satisfaction and motivation: Findings from a nationwide study in Portuguese higher education. Studies in Higher Education, 41(3), 541-559. doi:10.1080/03075079.2014.942265

Marqueze, E. C. e Moreno, C. R. C. (2009). Satisfação no trabalho e capacidade para o trabalho entre docentes universitários. Psicologia em Estudo, 14(1), 75-82. doi:10.1590/S1413-73722009000100010

Martinez, J. G. R. (1989). Cooling off before burnning out. Academic Therapy, 24(3), 271-284. doi:10.1177/105345128902400303

Mendes, F. (1996). A saúde e a doença dos professores universitários. Sociologia - Problemas e Práticas, (19), 165-187. Disponível em http://hdl.handle.net/10071/1051

Minter, R. L. (2009). Faculty burnout. Contemporary Issues in Education Research, 2(2), 1-8. Recuperado de https://files.eric.ed.gov/fulltext/EJ1056895.pdf

Olivas, O. L. L. e Martínez, R. E. G. (2010). Satisfacción laboral y percepción de salud mental en profesores. Revista Mexicana de Investigación en Psicología, 2(1), 33-38. Recuperado de http://revistamexicanadeinvestigacionenpsicologia.com/ojs/index.p hp/RMIP/article/viewFile/112/25

Organização Internacional do Trabalho. (1981). Informe de la reunión paritária sobre las condiciones de trabajo del personal docente. Genebra: Oficina Internacional del Trabajo. Recuperado de https://staging.ilo.org/public/libdoc/ilo/1982/82B09_315_span.pdf

Pessoa, F. S. (2015). Um olhar psicológico sobre fatores psicossociais de proteção e risco em contexto laboral (Dissertação de Mestrado, Universidade de Aveiro, Aveiro). Disponível em http://hdl.handle.net/10773/15589

Peterson, R. A. (1994). A meta-analysis of Cronbach's coefficient alpha. Journal of Consumer Research, 21(2), 381-391. doi: $10.2307 / 2489828$

Rodrigues, M. L. (1995). Atitudes da população portuguesa perante o trabalho. Organizações e Trabalho, (14), 33-63.

Schluter, H. L. e Valdéz, J. L. (2001). Situación del académico en las universidades mexicanas. Revista Latinoamericana de Psicología, 33(3), 261-267. Disponível em http://hdl.handle.net/20.500.11799/39878

Silva, C., Amaral, V., Pereira, A., Bem-Haja, P., Pereira, A., Rodrigues, V., ... Nossa, P. (2006). Copenhagen Psychosocial Questionnaire Portugal e Países Africanos de língual oficial portuguesa. Aveiro: Análise Exacta - Consultadoria, Formação e Edição de Livros.

Silva, T. A. N., Aquino, L. J., Fernandes, V. L. S., Zani, H. P., Evora, P. R. B. e Vento, D. A. (2016). Fatores de riscos para doenças cardiovasculares em docentes de ensino superior: Revisão de literatura. Revista Educação em Saúde, 4(1), 108-113. Recuperado de

http://revistas.unievangelica.com.br/index.php/educacaoemsaude/a rticle/view/1708

Sousa, I. F. e Mendonça, H. (2009). Burnout em professores universitários: Impacto de percepções de justiça e comprometimento afetivo. Psicologia: Teoria e Pesquisa, 25(4), 499-508. doi:10.1590/S010237722009000400005 
Souza, K. R., Mendonça, A. L. O., Rodrigues, A. M. S., Felix, E. G., Teixeira, L. R., Santos, M. B. M. e Moura, M. (2017). A nova organização do trabalho na universidade pública: Consequências coletivas da precarização na saúde dos docentes. Ciência \& Saúde Coletiva, 22(11), 3667-3676. doi:10.1590/1413812320172211.01192016
Vilela, E. F., Garcia, F. C. e Vieira, A. (2013). Vivências de prazersofrimento no trabalho do professor universitário: Estudo de caso em uma instituição pública. Revista Eletrônica de Administração, 19(2), 517-540. doi:10.1590/S1413-23112013000200010
C 2018 Lopes et al. This is an open access article distributed under the Creative Commons Attribution License, which permits unrestricted use, distribution, and reproduction in any medium provided the original work is properly cited. 